\title{
Tracheo-Innominate Artery Fistula after Stroke
}

\author{
Jong Hyun Mun, M.D., Po Sung Jun, M.D., Young-Joo Sim, M.D. ', \\ Ho Joong Jeong, M.D. ${ }^{1}$, Ghi Chan Kim, M.D. ${ }^{1}$ \\ Department of Physical Medicine and Rehabilitation, Dong-Eui Hospital and \\ ${ }^{1}$ Kosin University College of Medicine, Busan 602-702, Korea
}

Tracheo-innominate artery fistula (TIAF) is rare, yet the most fatal complication after tracheostomy. In the absence of immediate diagnosis and surgical management, the mortality rate is very high, because the complication can lead to sudden massive tracheal hemorrhage. Tracheal obstruction and hypovolemic shock are the major life threatening conditions. The 46-year-old woman received tracheostomy tube insertion after stroke. Three months later, there was occurrence of active bleeding at the site of tracheostomy in the patient, who participated in comprehensive rehabilitation program. Immediately, the patient received an endotracheal tube insertion into the tracheostomy site and thus massive bleeding was controlled. The patient was transferred to the intensive care unit, where her breathing was maintained by mechanical ventilation. Based on computed tomography and laryngoscopy, no remarkable findings about TIAF were detected. Nevertheless, transfemoral angiography findings revealed that innominate artery made small luminal outpouching to trachea at the carotid artery and at the subclavian artery bifurcation level, based on which a diagnosis of TIAF was made. She had an operation for TIAF, tracheoplasty with bypass graft. Subsequently, she was discharged after 15 weeks. In the present report, we describe a case of TIAF, which can occur in the patients with tracheostomy tube during rehabilitation.

Key Words Trachea, Innominate artery, Fistula

\section{INTRODUCTION}

Tracheo-innominate artery fistula is rare but fatal complication that may occur after a tracheotomy. Potential

Received December 16, 2010; Accepted January 31, 2012

Corresponding author: Ghi Chan Kim

Department of Physical Medicine and Rehabilitation, Kosin University College of Medicine, 34, Amnam-dong, Seo-gu, Busan 602-702, Korea

Tel: +82-51-990-6156, Fax: +82-51-990-3181, E-mail: ghckim@hanmail. net

(c) This is an open-access article distributed under the terms of the Creative Commons Attribution Non-Commercial License (http:// creativecommons.org/licenses/by-nc/3.0) which permits unrestricted noncommercial use, distribution, and reproduction in any medium, provided the original work is properly cited.

Copyright $\odot 2012$ by Korean Academy of Rehabilitation Medicine tracheo-innominate artery fistula must be inspected upon the occurrence of massive hemorrhage at trachea. Most of the patients die unless appropriate initial treatment is provided so that the appropriate initial treatment method is important. Physiatrists should especially have thorough knowledge of the potential complication and relevant treatment since they frequently take care of patients with tracheotomy. This study investigates relevant researches along with a specific case report.

\section{CASE REPORT}

A 46-year-old female had a tracheotomy under diagnosis of pneumonia during her course of conservative treatment (Fig. 1) after being hospitalized in a neurosurgery 


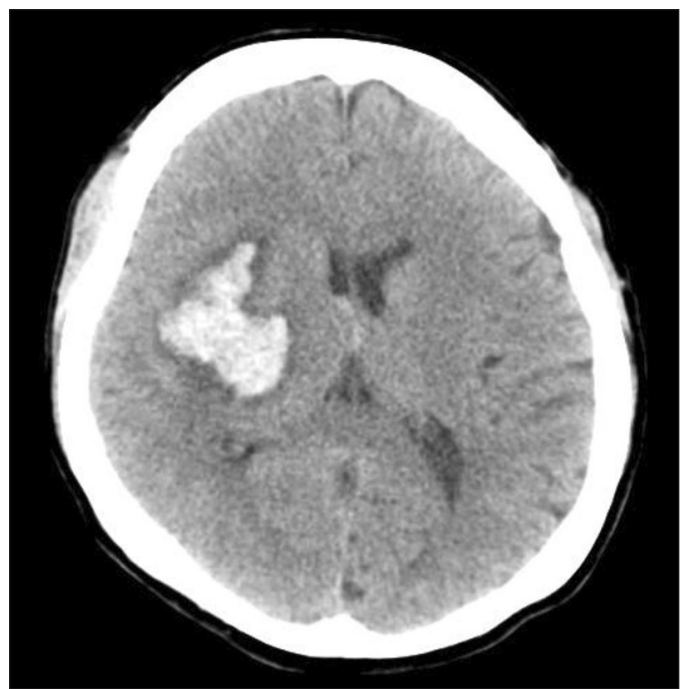

Fig. 1.Non-contrast brain CT demonstrating bleeding on right basal ganglia.

center for intracerebral bleeding at the right basal ganglia region. After two months of the intracerebral hemorrhage, she was transferred to a rehabilitation center. In the third month after the tracheotomy, massive hemorrhage occurred through the tracheotomy tube when tracheal suction was carried out. The patient demonstrated cyanosis, respiratory failure, and shocks so that the cuff air in tracheotomy tube was maximally expanded. However, the bleeding did not stop. The tube was immediately removed while the suction was consistently carried out. A cuffed endotracheal intubation was tried through the oral cavity but the attempt was a failure due to visual obstruction. The cuffed endotracheal tube was inserted deep into the tracheal opening, and the cuff was expanded, followed by moving up the tube. The bleeding stopped at last. She was immediately transferred to an intensive care center, and blood test was carried out. The hemoglobin level decreased from $10.9 \mathrm{~g} / \mathrm{dl}$ to $8.9 \mathrm{~g} / \mathrm{dl}$ (about a drop of $2 \mathrm{~g} / \mathrm{dl}$ ), and cervical CT was carried out to identify the cause of bleeding. No special abnormality was identified, but the bronchoscopy did not show endotracheal area due to blood clot. Complete examination could not be done due to the risk of re-bleeding, hence conservative treatment was continued. One week after the bleeding, transfemoral angiography findings revealed that innominate artery made small luminal outpouching to trachea (Fig. 2) at the carotid artery and subclavian artery bifurcation level, and thus diagnosis of TIAF was made. She was transferred to the thoracic surgery unit,

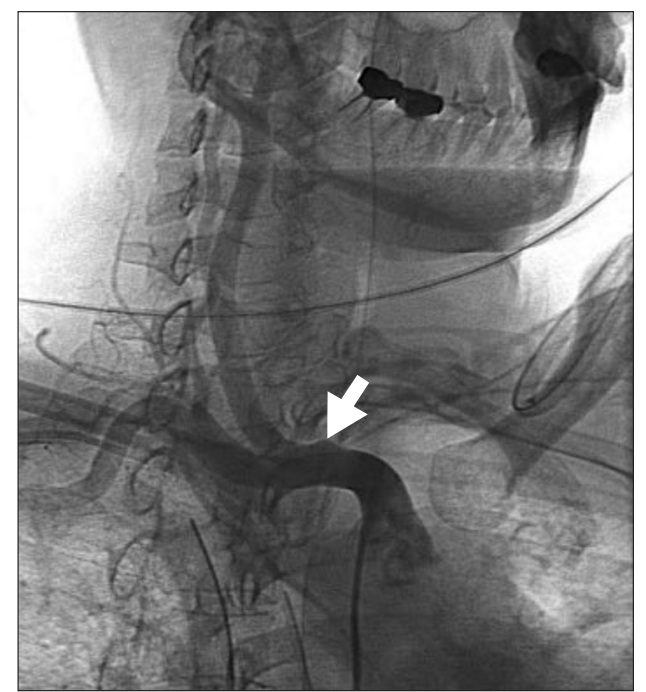

Fig. 2. Small luminal outpouching to innominate artery (arrow) in chest subclavian angiography.

and had an operation for TIAF, tracheoplasty with bypass graft. A rupture area of $1 \times 0.5 \mathrm{~cm}$ was observed by a median sternotomy, and the innominate artery was rebuilt through replacement of the detached innominate artery by an artificial blood vessel that connected the right subclavian artery and the right aorta while the tracheoplasty was done using the fifth costal cartilage. A month later, tracheostenosis and inflammation were observed during a bronchoscopy that was carried out due to wheezing and tachypnea and subsequently, endotracheal balloon dilatation was conducted. However, no improvement in the symptoms was seen, hence replacement of Montgomery T-tube was carried out followed by continuation of rehabilitation after transferring her to the rehabilitation center. It has been eleven months since the surgery has been carried out, and the patient survives without further complications.

\section{DISCUSSION}

Tracheo-innominate artery fistula was reported in 1879 for the first time by Korte in the case of a pediatric patient, a five years old diphtheria patient. The incidence of tracheo-innominate artery fistula after tracheotomy has been reported between $0.3 \%$ and $0.7 \%$. And most often, it occurs between third to twenty fourth days after the surgery though it may occur anytime during and after the surgery. However, it occurs frequently even several years after tracheostomy due to the developed management of 
tracheostomy, and it has become the most critical complication of the late stage. ${ }^{1}$ The cause of death is rather apparent death due to aspiration of blood than loss of blood. The condition is extremely fatal in the absence of appropriate emergency treatment. Even with the surgical treatment, the mortality rate is reported to be $73 \%$. The most frequent mechanism of tracheo-innominate artery fistula is known as the pressure necrosis of the inner wall of trachea and the mechanical erosion, and it can be further divided mechanically into cases of the direct damage by the concave portion of the tube, damages caused by the cuff of tracheotomy tube, and direct stimulation of cannula tip portions of tracheotomy tube. The condition progresses to necrosis with consequent damage to tracheal cartilages and invasion into innominate artery. Sepsis, radiation therapy, stoma infection, steroid therapy and malnutrition also contribute towards tracheal mucosal injury. Other than on the innominate artery, fistulas may occur on the common carotid artery, the innominate vein, the inferior thyroid artery, the internal mammary artery, or the aortic arch. Risk factors include long-term mechanical ventilation, high ventilator peak pressures, low-lying tracheostomy sites typically below the third tracheal ring, ${ }^{2,3}$ and previous tracheal resections. Fistula formation has been attributed to high cuff pressures, and it is recommended to maintain the pressure in the tracheostomy cuff to be less than $20 \mathrm{mmHg}{ }^{4}$ Since the tracheo-innominate artery fistula is extremely fatal, preventive measures are the most important aspect. Appropriate humidity, clean maintenance, infection management, and smooth tracheal suction should be ensured, and excessive extension of neck needs to be prevented. Special attention should be paid in the case of patients with short neck or children, and appropriate size of the tracheotomy tube needs to be selected. Excessive movements also need to be prevented for patients with head injuries. Nevertheless, the most important preventive measure is early detachment of the artificial respirator. Moreover, appropriate treatment and management are required for patients having tracheal granuloma. ${ }^{5}$ If serious bleeding occurs at the tracheostomy site, be suspicious of the tracheo-innominate artery fistula, and be sure to be fully aware of appropriate management. Typically, $65 \%$ of patients with the tracheo-innominate artery fistula show acute large amount of bleeding, but $35 \%$ of them show only minor bleeding. However, minor bleeding and pulsating movement of the tracheotomy cannula are preceded 2 hours to 3 days before the occurrence of serious bleeding in the case of 35 to $50 \%$ of the patients, ${ }^{6}$ hence the potential tracheo-innominate artery fistula should be considered if a bleeding of $10 \mathrm{ml}$ or more is observed within 48 hours after the tracheotomy. When massive hemorrhage occurs from trachea, controlling the hemorrhage and securing the airway are the most important things to do since the risk of death becomes large due to respiratory tract obstruction, respiratory failure, and hypovolemic shock. Homeostasis should be tried by applying pressure on the bleeding area by overinflation of the tube cuff. The tracheostomy tube needs to be removed immediately if the bleeding continues, followed by oropharyngeal intubation with a cuffed oral endotracheal tube. The balloon cuff should be excessively expanded from the lower portion of the bleeding area to prevent accumulation of blood in the lungs. Subsequently, stomal hemorrhage gets controlled by digit compression of the innominate artery against the manubrium. This procedure involves penetration of the pretracheal fascial plane through the tracheostomy wound. The innominate artery is then bluntly dissected off the trachea with the index finger and compressed against the manubrium. This maneuver can also be accomplished by placing a finger through the stoma and into the trachea itself alongside the endotracheal tube. Distal compression has been reported to be successful in about $90 \%$ of cases, and the pressure should be continued during transfer of the patient to the operating room. However, the homeostasis does not work with this method, overinflation of the balloon cuff at the bleeding portion should be repeatedly carried out by adjusting the position of oral endotracheal tube up and down. The patient should be transported to an operation room immediately after the bleeding stops, and rigid bronchoscopic examination of the trachea needs to be carried out even after the surgery is prepared since re-bleeding may occur during tracheal bronchoscope. ${ }^{5}$ If any evidence of hemorrhage, necrosis, and ulcer is identified, the condition becomes the indication for surgery. Angiography may be a diagnostic option but is hard to be carried out due to lack of time. Even if it is carried out, the result may be normal since the bleeding at the time of test is likely to be ceased already. ${ }^{7} \mathrm{~A}$ full median sternotomy has traditionally been the most common approach for the repair of TIAF. Ligation of the innominate artery or resection of a segment of the innominate artery and oversewing of the ends is known 
to have better survival rate than interposition grafting or direct vascular repair by suturing. In our case, the innominate artery was rebuilt through replacement of the detached innominate artery by an artificial blood vessel that connects the right subclavian artery and the right aorta while the tracheoplasty was done using the fifth costal cartilage. Though no domestic research has been conducted on the tracheo-innominate artery fistula after stroke, possible increase in damage to trachea by repetitive opisthotonos for patients with head trauma have been reported. According to the research by Hamano et al., ${ }^{5}$ evidence of ulceration was noted in the case of all the patients; 10 TIAF patients with severe motor and intellectual disability (SMID), while seven of them had the history of epileptic convulsion. Moreover, according to the report by Thorp et al., ${ }^{8}$ the neurological spasticity with abnormal arching of the neck might have contributed towards the formation of the TIAF, resulting in increased pressure from the cannula against the anterior tracheal wall. This report describes our experience with an unknown case of tracheo-innominate artery fistula in rehabilitation unit. Since ample studies are not available on tracheo-innominate artery fistula for stroke patients, preventive measures should be taken for patients with tracheotomy. These measures should be observed frequently in the rehabilitation unit, including appropriate posture, restriction of excessive movements, continuous management of tracheal tubes, and sustained management of erosion and ulceration in the trachea to reduce the pressure between the anterior wall of trachea and the tracheal tube. In case of hemorrhage occurring after tracheotomy, there might be fatal consequences despite of low frequency. Hence, thorough knowledge of appropriate treatment for the tracheo-innominate artery fistula is warranted along with prompt execution of treatment.

\section{REFERENCES}

1. Epstein SK. Late complications of tracheostomy. Respir Care 2005; 50: 542-549

2. Deguchi J, Furuya T, Tanaka N, Nobori M, Seki Y, Nomura Y, Umehara I, Saito H, Miyata T. Successful management of trachea-innominate artery fistula with endovascular stent graft repair. J Vasc Surg 2001; 33: $1280-1282$

3. Black MD, Shamji FM, Todd TR. Trachea-innominate artery fistula and concomitant critical cerebrovascular disease. Ann Thorac Surg 1996; 62: 286-288

4. Kirby RR. The monitoring of mechanically ventilated patients. Int Anesthesiol Clin 1997; 35: 65-86

5. Hamano K, Kumada S, Hayashi M, Uchiyama A, Kurihara E, Tamagawa K, Enomoto S, Chou H. Hemorrhage due to tracheoarterial fistula with severe motor and intellectual disability. Pediatr Int 2008; 50: 337 340

6. Singh N, Fung A, Cole IE. Innominate artery haemorrhage following tracheostomy. Otolaryngol Head Neck Surg 2007; 136: S68-72

7. Bloss RS, Ward RE. Survival after tracheoinnominate artery fistula. AM J Surg 1980; 139: 251-253

8. Thorp A, Hurt TL, Kim TY, Brown L. Tracheoinnominate artery fistula: a rare and often fatal complication of indwelling tracheostomy tubes. Pediatr Emerg Care 2005; 21: 763-766 